\title{
ESCORRENTIA Y EROSION EN LOS SUELOS DEL DESIERTO DE JUDEA
}

\author{
Artemi CERDA \\ Fysisch Geografisch en Bodemkundig Laboratorium \\ Universiteit van Amsterdam. Paises Bajos \\ Hanoch LAVEE \\ Department of Geography \\ Bar-llan University. Israel
}

\begin{abstract}
Resumen: Los suelos del Desierto de Judea presentan unas elevadas tasas de escorrentía fruto de encharcamientos e inicios de escorrentía muy rápidos ( 1 ' 13" y $2^{\prime} 03$ " respectivamente), tasas de infiltración final estable muy reducidas ( $7 \mathrm{~mm}$ $\mathrm{h}^{-1}$ ) y curvas de escorrentía de gran pendiente, alcanzándose la estabilidad de las tasas de escorrentía antes de los 20 minutos.

Las tasas de erosión son elevadas debido a los grandes volúmenes de escorrentía y a la elevada erosionabilidad de los suelos, afectados por el pastoreo desde hace milenios. En cambio, la salinidad de la escorrentía es insignificante en relación con las sales acumuladas en los suelos.

Los suelos menos afectados por el pastoreo (Ma'ale Adumin) generan elevados volúmenes de escorrentía, aunque la carga sólida es ínfima. Esto se debe al efecto positivo de las costras superficiales y a la abundancia de fragmentos de roca en la reducción de la erosionabilidad de los suelos. Los suelos más afectados por el pastoreo (Mishor Adumin) presentan costras alteradas y polvo depositado en las superficies, lo que da lugar a concentraciones de sedimentos en la escorrentía de hasta $10 \mathrm{~g} \mathrm{l}^{-1}$, disparando las tasas de erosión hasta más de $400 \mathrm{~g} \mathrm{~m}^{2} \mathrm{~h}^{-1}$, y los coeficientes de escorrentía hasta 0,90 .

Los resultados demuestran la gran importancia de las costras biológicas sobre la hidrología y erosionabilidad de los suelos en ambientes semiáridos, así como la posibilidad de escorrentía superficial directa -hortoniana- durante las tormentas de elevada intensidad. El uso antrópico del suelo, en este caso el pastoreo, afecta los mecanismos de transformación de la lluvia en escorrentía, y con ello favorece el aumento de las tasas de erosión.
\end{abstract}

Palabras clave: Desierto, lluvia-simulada, costras, Hidrología, erosión. 


\begin{abstract}
The Judean Desert soils are characterised by high runoff rates, short times to ponding and runoff ( $11^{\prime} 13^{\prime \prime}$ and $2^{\prime} 03^{\prime \prime}$ respectively), low steady state infiltration rates $\left(7 \mathrm{~mm} \mathrm{~h}^{-1}\right)$ and very high angles of runoff curves, reaching the stability of the runoff rates earlier than 20 minutes.

The erosion rates are very high, shown by the large volumes of runoff collected due to the high erodibility of soils, affected by the grazing during millenniums. On the contray, the salinity of the runoff is very low in relation with the salts accumulated in the soils.

The soils less affected by grazing (Ma'ale Adumin), produce large runoff volumes, although the milkeness was very low. This is due to the positive effect of the surficial crust and to the abundance of rocks fragments in the reduction of the erodibility of the soils. The soils more affected by grazing (Mishor Adumin) show disturbed crusts and dust accumulation on the surfaces, causing higher runoff sediment concentrations (even $10 \mathrm{~g} \mathrm{l}^{-1}$ ), erosion rates $\left(400 \mathrm{~g} \mathrm{~m}^{2} \mathrm{~h}^{-1}\right.$ ) and runoff coefficients $(0,90)$ to ocurr.

The results demonstrate the importance of the biological crust for the hydrology and erodibility of soils in semiarid environments, and for the posibility of the surface -hortonian- overland flow during the high intensity thunderstorms to ocurr. The anthropic use of the land, in this case grazing, modifies the mechanisms that transform the rainfall into runoff, and induce erosion to ocurr.
\end{abstract}

Key words: Desert, simulated-rain, crusts, Hydrology, erosion.

\title{
INTRODUCCION
}

En los climas con bajas precipitaciones los suelos presentan un escaso desarrollo y una cubierta vegetal insignificante. Una implicación geomorfológica de estas condiciones es la importancia de la erosión eôlica, si bien los desiertos arenosos son minoritarios (COOKE et al., 1993). Las zonas áridas, además de los escasos volúmenes de precipitación, presentan elevadas tasas de evapotranspiración (COOKE \& REEVES, 1972). La lluvia se restringe a unos pocos días al año y su heterogeneidad espacial es muy elevada. Un elevado porcentaje de las precipitaciones es fruto de tormentas convectivas, y la irregularidad temporal es tan alta que en ocasiones las precipitaciones diarias superan las lluvias anuales totales (SLAYTER \& MABBUT, 1964; SHICK, 1987).

Por todo ello, además del viento, en las zonas desérticas el agua es un agente modelador de primera magnitud. Esto se debe a que las lluvias, aunque escasas, son intensas y a que los suelos están pobremente protegidos por la vegetación, lo que da lugar a una gran actividad de algunos procesos geomorfológicos superficiales (YAIR \& LAVEE, 1985). A pesar de la importancia de los procesos de erosión fluvial en las 
zonas áridas y semiáridas existe poca información sobre ellos. Se desconocen los factores, los mecanismos básicos de transformación de la lluvia en escorrentía y existen actualmente muy pocas mediciones de procesos básicos como son la infiltración, la generación de escorrentía y la pérdida de suelo (THORNES, 1994).

Parece obvio que si en las zonas desérticas o semidesérticas las lluvias son muy intensas y los suelos stàn poco desarrollados la arroyada superficial debe ser el proceso de mayor impacto geomorfológico (LEOPOLD et al., 1966; SCHICK, 1977). Experimentos similares a los aquí presentados han demostrado escorrentías elevadas y concentraciones de sedimentos reducidas en la zona más árida del Desierto de Judea, junto al mar Muerto (CERDA \& LAVEE, 1994) y en zonas con mayores volúmenes de precipitación (LAVEE et al., 1991), similares a las aquí estudiadas, pero siempre dentro del desierto de Judea.

El objetivo de este trabajo es aportar información sobre la generación de escorrentía y pérdida de suelo ante lluvias intensas en la zona oeste del Desierto de Judea, donde las precipitaciones anuales varían entre 260 y $330 \mathrm{~mm}$.

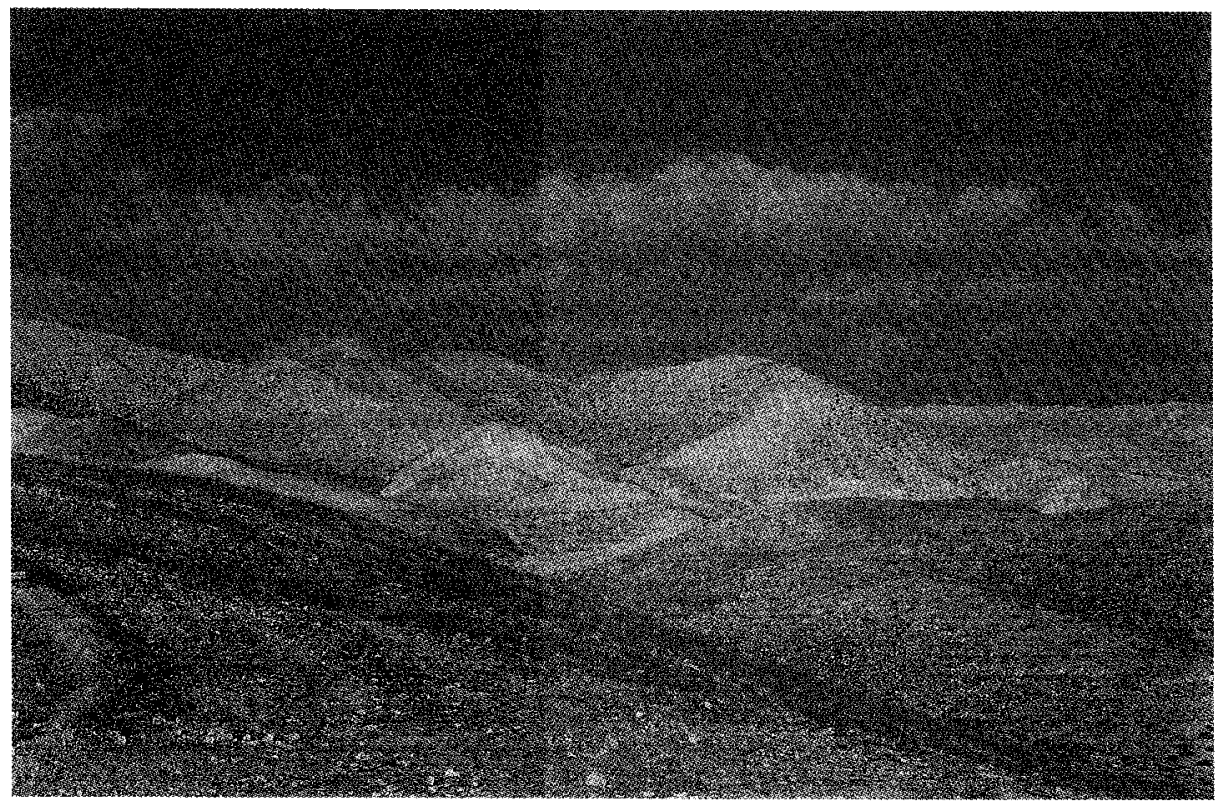

Figura 1.- Vista de la zona de estudio de Mishor Adumin. 


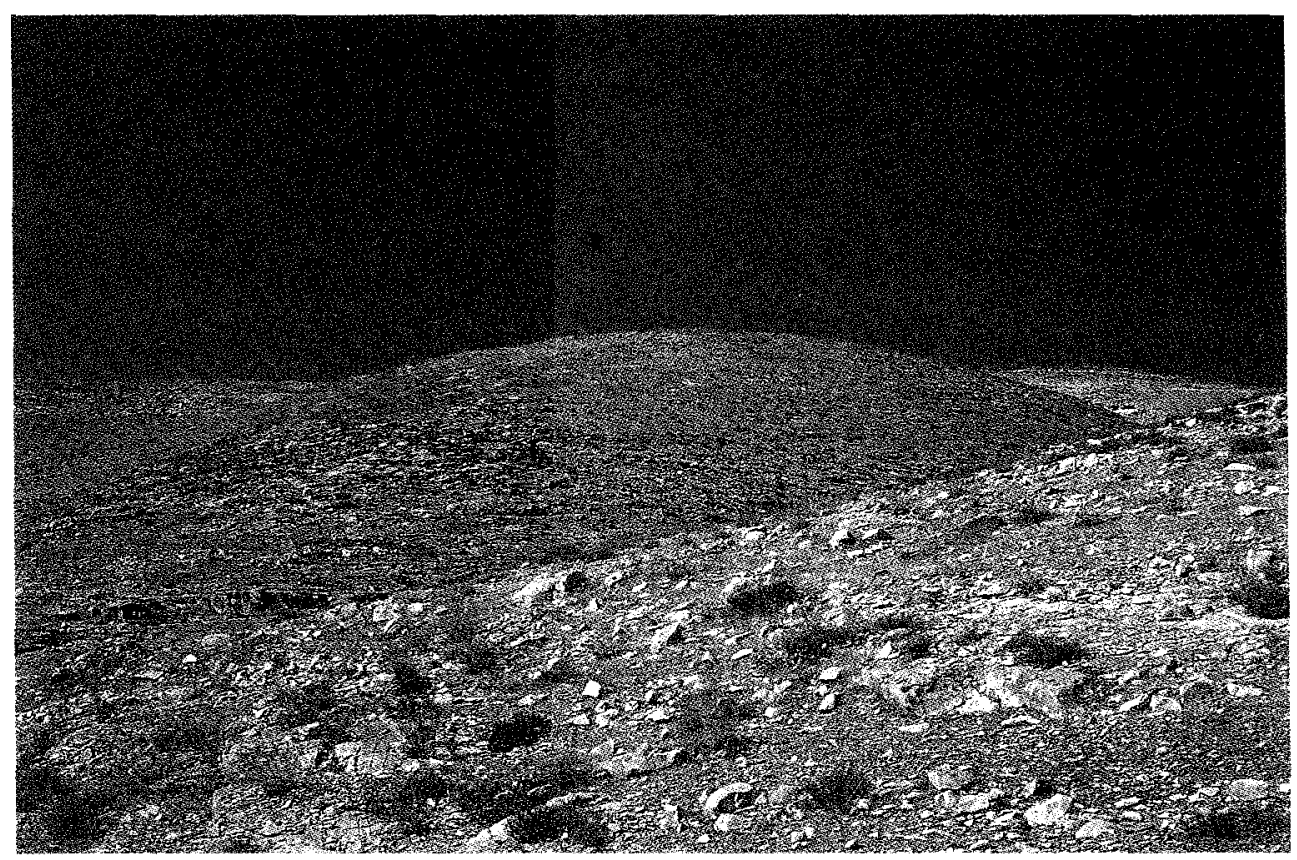

Figura 2.- Vista de la zona de estudio de Maíale Adumin.

\section{AREA DE ESTUDIO}

El desierto de Judea comprende una franja de unos $25 \mathrm{~km}$ de anchura entre el mar Muerto y Jerusalén. Hay un claro gradiente climatológico de norte a sur debido a la latitud, y de oeste a este por la altura y lejanía al mar Mediterráneo. En el primer caso la reducción de las precipitaciones y el aumento de las temperaturas y tasas de evapotranspiración son evidentes hacia el sur, pero los cambios son poco marcados. La zona sur del desierto de Judea -la más árida- entra en contacto sin solución de continuidad con el desierto del Negev. En cambio, la reducción de la altura desde las montañas de Jerusalén $(650 \mathrm{~m})$ hasta el mar Muerto $(<400 \mathrm{~m})$ supone cambios drásticos en las precipitaciones de oeste a $(620 \mathrm{~mm})$ a este $(100 \mathrm{~mm}$ anuales) (LAVEE et al., 1994). De este gradiente altitudinal y climático se han seleccionado dos zonas de estudio: Mishor Adumin (Fig. 1) y Ma'ale Adumin (Fig. 2), las cuales son representativas de la zona oeste del desierto de Judea (Fig. 3). Allí, el paisaje está caracterizado por una muy baja cubierta vegetal $(10 \%)$, laderas con pendientes entre $5-15^{\circ}$, suelos poco desarrollados y cubiertos por fragmentos de roca. La litología está 
dominada por las calizas, aunque también se encuentran margas en la zona más cercana al mar Muerto, sobre las que se desarrolla un paisaje de badlands. En cada una de las zonas de estudio se seleccionó una ladera de orientación sur y pendientes medias de $10^{\circ}$ donde se construyeron seis parcelas de $0,24 \mathrm{~m}^{2}$ en superficies representativas. Al contrario que sobre litologías blandas, en las laderas sobre calizas del desierto de Judea no existen regueros, pero sí encostramiento superficial de carácter biológico, físico y/o químico y una abundante cubierta de fragmentos de rocas.

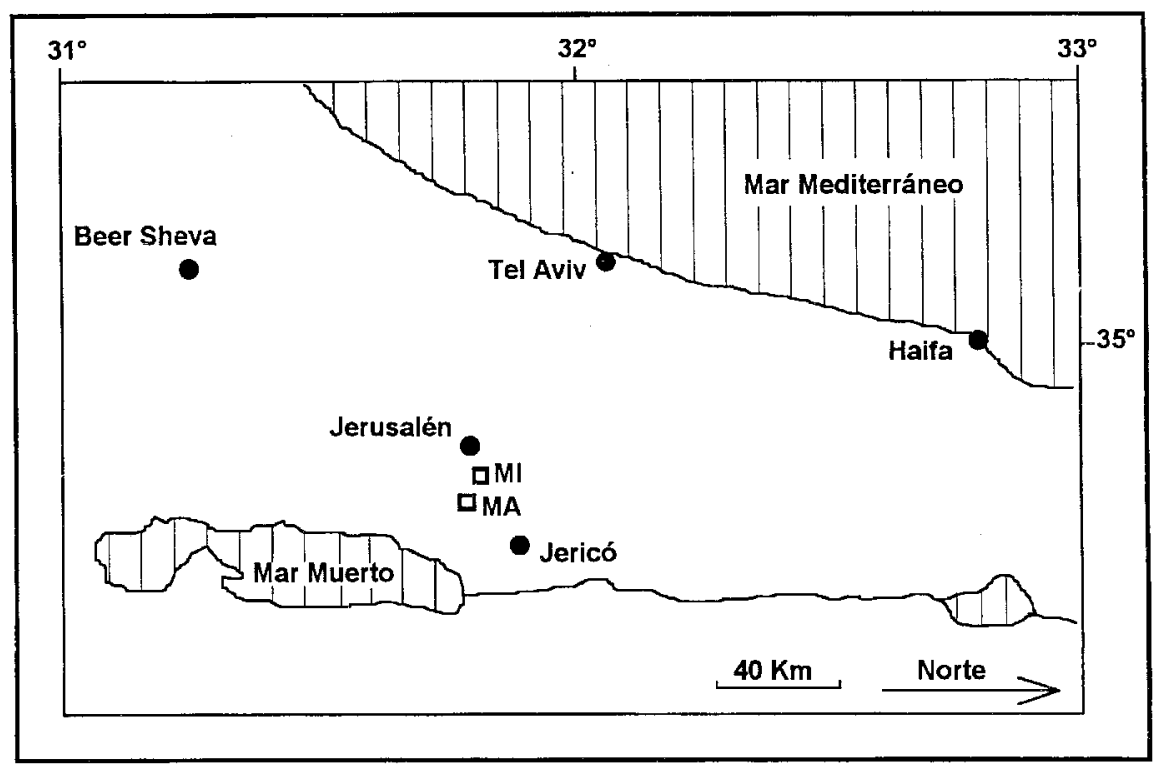

Figura 3.- Localización de las zonas de estudio. MI, Mishor Adumin y MA, Ma'ale Adumin.

El clima es semiárido $(260-330 \mathrm{~mm})$. El invierno es la estación más húmeda, aunque también pueden producirse chaparrones convectivos durante el verano. La temperatura media anual es de $19-20^{\circ}$ y las heladas nocturnas son prácticamente inexistentes.

La vegetación está compuesta básicamente por pequeños matorrales y herbáceas. Además de una cubierta de fragmentos de roca y costras de origen físico o químico hay un desarrollo importante de cubierta de líquenes, algas y musgos. A pesar de la reducida distancia $(5 \mathrm{~km})$ y la similitud del clima, la composición florística es muy distinta en cada zona de estudio. Las especies dominantes en Ma'ale Adumin son 
Sarcopoterium spinosum, Phlomis Brachodo, Filago sp., Plantago coronopus y Asphodelous microcarpus, mientras que en Mishor Adumin son Anabasis articulata, Halogetan alopecuroides, Salsola vermiculata, Reamuria hirtella y Anagalis arvensis.

La cubierta vegetal en ambas zonas de estudio es mas reducida de lo esperado para precipitaciones anuales que alcanzan los $300 \mathrm{~mm}$, lo que sin duda está directamente relacionado con el sobrepastoreo de la zona desde hace milenios. Aunque en todo el desierto de Judea la presión del pastoreo es muy alta, en Mishor Adumin alcanza su máxima expresión, lo que explica que la cubierta vegetal sea muy baja y que la composición florística sea distinta.

El área donde se ha realizado el estudio forma parte de una amplio proyecto de investigación, en el cual se están estudiando los mismos tópicos a escala de ladera con parcelas de erosión (LAVEE et al., 1994). Estos experimentos vienen a completar la información existente.

Las reducidas modificaciones de las parcelas son debidas a la topografía de la ladera, aunque todas las parcelas fueron seleccionadas en la parte media de ésta con el fin de eliminar variables que influyan en el comportamiento hidrológico y erosivo y hacer que los resultados sean comparable con otros estudios (CERDA \& LAVEE, en prensa). La textura del suelo es franca y la estabilidad de los agregados es reducida, especialmente en la zona de Mishor Adumin donde en ninguna época del año superan los agregados 40 impactos de gota. Respecto a la agregación, en Ma'ale Adumin hay diferencias según las estaciones, siendo en invierno 95 el número medio de gotas necesarias para la desagregación de los agregados. Esta diferencia se debe básicamente a que en Mishor Adumin el contenido en materia orgánica es muy bajo, $2,3 \%$ en superficie y $2 \%$ a $6 \mathrm{~cm}$ de profundidad, mientras que en Ma'ale Adumin es de 4 , 2 y 2,6\% respectivamente. El pH es de 7,35-7,45 en Mishor Adumin y de 7,2-7,3 en Ma'ale Adumin. La conductividad eléctrica del suelo (pasta saturada) es de 1,6 y 1,3 en Mishor Adumin y de 1,37 y 0,64 $\mathrm{mmoh} \mathrm{cm}^{-1}$ en Ma'ale Adumin para las mismas profundidades citadas anteriormente (LAVEE et al., 1994).

\section{MATERIAL Y METODOS}

Los experimentos consistieron en chaparrones de una intensidad de $50,36 \mathrm{~mm} \mathrm{~h}^{-1}$ mantenida durante 45 minutos. En todos los casos, se pretendía alcanzar la infiltración final estable con el fin de conocer este parámetro, así como la curva de escorrentía en su totalidad. El caudal se midió a intervalos de un minuto y al menos cada 10' se tomaron muestras con el fin de conocer la concentración de sedimentos de la arroyada, la salinidad y las tasas de erosión. 


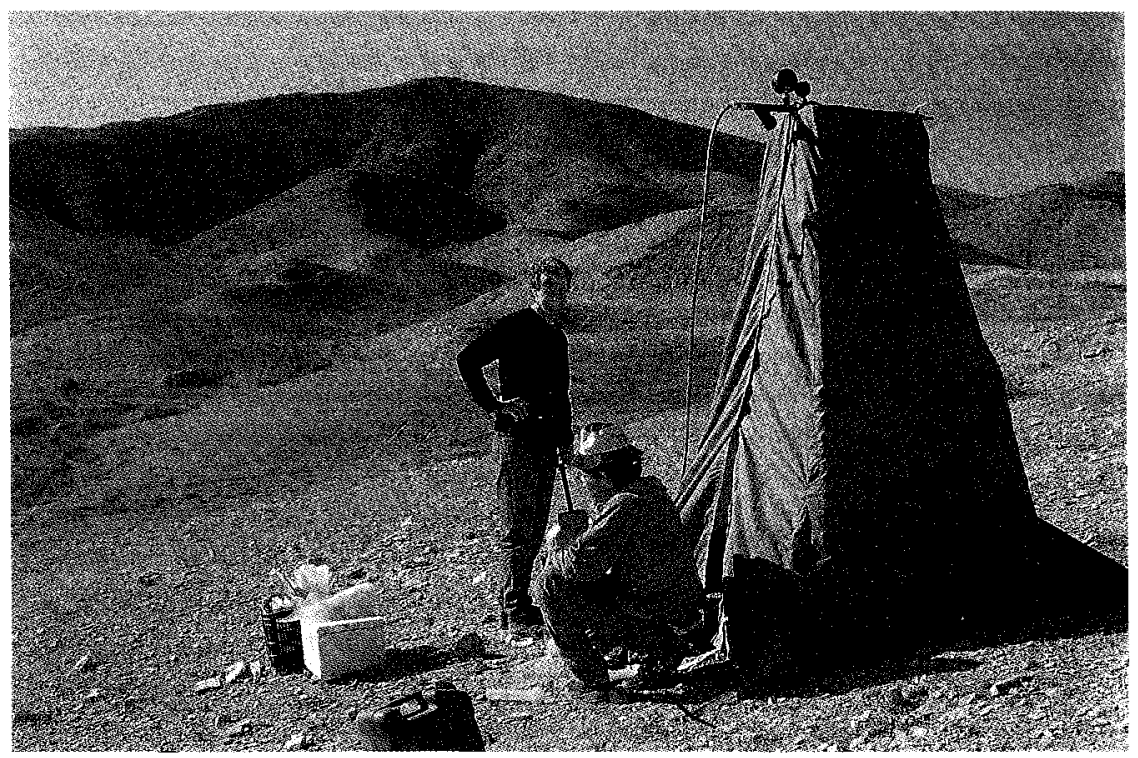

Figura 4.- Vista del simulador de lluvia.

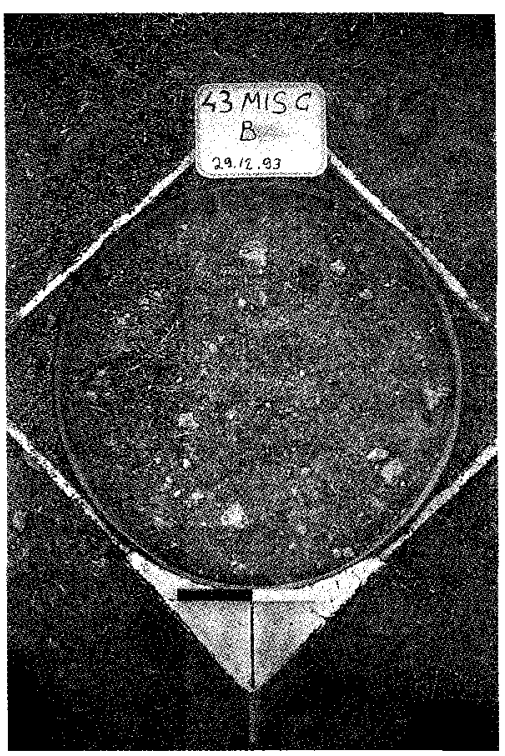

Figura 5.- Vista de una de las parcelas. 
En cada parcela se han medido al menos cuatro veces estas variables a intervalos regulares. La conductividad eléctrica de la escorrentía se midió directamente en el campo tras su filtrado. Ello permite conocer la salinidad de la escorrentía en distintas fases del hidrograma. Las variaciones temporales de la concentración de sedimentos (TCS) y la conductividad eléctrica (TCE) se han modelizado mediante el ajuste a una ecuación del tipo $\mathrm{y}=\mathrm{a}+\mathrm{b}(\mathrm{x})$ de los valores obtenidos en el campo, mostrando el "parámetro b" la tendencia positiva o negativa de estas variables. Una detallada explicación de la metodología y el tratamiento de los datos se puede encontrar en CERDA (1993a; 1993b;1995).

El simulador de lluvia utilizado es del tipo pulverizador, siendo el tamaño de las parcelas de $0,24 \mathrm{~m}^{2}$, aunque el área humectada es superior a $1 \mathrm{~m}^{2}$ (ver figuras 4 y 5). La lluvia simulada siempre se ha producido con agua desionizada debido a la importancia de las sales en la generación de la escorrentía en este tipo de suelos (SHAINBERG et al., 1981; IMESON y VERSTRATEN, 1988). Al final de los experimentos se miden los frentes de humedad y se toman muestras de suelo con el fin de determinar la humedad del suelo a dos profundidades: 0-2 y 4-6 cm. Antes del inicio del experimento también se toman muestras para conocer la situación previa.

Los experimentos se realizaron durante de el mes de diciembre de 1993 inicio de la época de lluvias. La curva de escorrentía medida en el campo ha sido modelizada mediante la ecuación de HORTON (1940):

$$
i=f c-(f o-f c) e^{\alpha t}
$$

Donde: $\quad i=$ tasa de infiltración instantánea

$\mathrm{fc}_{\mathrm{c}}=$ infiltración final o constante

fo = infiltración inicial

$\mathrm{t}=$ tiempo

$\alpha=$ decaimiento exponencial de la tasa de infiltración instantánea

En cada una de las dos zonas de estudio la estrategia de muestreo consistió en la selección de seis superficies representativas: MI01, MI02, MI03, MI04, MI05 y MI06 en Mishor Adumin y MA01, MA02, MA03, MA04, MA05 y MA06 en Ma'ale Adumin. 
Tabla 1.- Características de las superficies estudiadas: Exposición (Exp), Pendiente (Pen), cubierta de Líquenes (Liq), Musgos (Mus) y Plantas (Pla), Altura de la vegetación (Ave), cubierta de Fragmentos de rocas (Pie) y Profundidad del suelo (Prs).

\begin{tabular}{|c|c|c|c|r|r|r|r|c|}
\hline PARCELA & Exp & Pen & Liq & Mus & Pla & Alv & Pie & Prs \\
\hline & $\left({ }^{\circ}\right)$ & $\left(^{\circ}\right)$ & $(\%)$ & $(9)$ & $(\%)$ & cm & $(\%)$ & cm \\
\hline MI01 & 180 & 2 & 0 & 0 & 0 & 0 & 60 & 25 \\
\hline MIO2 & 185 & 10 & 0 & 0 & 0 & 0 & 65 & 24 \\
\hline MI03 & 185 & 4 & 0 & 0 & 0 & 0 & 25 & 4 \\
\hline MI04 & 125 & 9 & 25 & 45 & 2 & 1 & 5 & 23 \\
\hline MI05 & 175 & 5 & 80 & 0 & 1 & 0,5 & 20 & 10 \\
\hline MI06 & 180 & 3 & 80 & 0 & 10 & 3 & 20 & 12 \\
\hline MA01 & 180 & 10 & 30 & 30 & 5 & 1 & 30 & 35 \\
\hline MA02 & 190 & 7 & 20 & 20 & 5 & 1 & 25 & 25 \\
\hline MA03 & 180 & 3 & 5 & 3 & 2 & 1 & 70 & 30 \\
\hline MA04 & 185 & 8 & 2 & 0 & 20 & 5 & 80 & 23 \\
\hline MA05 & 190 & 10 & 1 & 0 & 5 & 3 & 80 & 21 \\
\hline MA06 & 186 & 8 & 30 & 30 & 20 & 7 & 20 & 26 \\
\hline Media & 178,4 & 6,6 & 22,7 & 10,7 & 5,8 & 1,8 & 41,7 & 21,5 \\
\hline
\end{tabular}

\section{RESULTADOS}

Los suelos del desierto de Judea, litosoles, presentan una elevada superficie cubierta de piedras $-42 \%$ de media en las parcelas estudiadas- que alcanza puntualmente valores hasta el $80 \%$. Todas los datos presentados se refieren a las cubiertas de las parcelas.

Los suelos seleccionados para los experimentos muestran profundidades entre $4 \mathrm{y}$ $35 \mathrm{~cm}$, y en general la cubierta de plantas es muy baja (0-20\%), aunque los líquenes -hasta el $80 \%$ - y los musgos -hasta el $45 \%$ - son muy abundantes. Es importante destacar que nunca aparecen superficies totalmente desnudas, y líquenes y musgos, 0 piedras protegen el suelo del impacto directo de las gotas de lluvia. A pesar de ello, puntualmente aparecen pequeñas superficies que exponen la matriz del suelo a la atmósfera, y cuando ocurre siempre existe una costra superficial -física y/o químicaque presenta pequeñas grietas $(1 \mathrm{~mm})$. Las costras biológicas -líquenes y musgos- no muestran agrietamientos, o éstos son muy reducidos. 
Tabla 2.- Humedad del suelo antes y después de los experimentos a dos profundidades: 0-2 cm y 4-6 cm. Tiempo de encharcamiento (tp), de escorrentía en superficie (ts), de su medición en la salida de la parcela (te) y volumen de lluvia necesario para producir escorrentía fuera de la parcela (Vte).

HUMEDAD DEL SUELO (\%)

\begin{tabular}{|c|c|r|c|c|c|c|c|l|}
\hline & \multicolumn{2}{|c|}{ ANTES } & \multicolumn{2}{c|}{ DESPUES } & tp & ts & te & Vte \\
\hline PARCELA & $(0-2 \mathrm{~cm})$ & $(4-6 \mathrm{~cm})$ & $(0-3 \mathrm{~cm})$ & $(4-6 \mathrm{~cm})$ & min. & min. & min & mm. \\
\hline MI01 & 4,26 & 5,73 & 18,12 & 5,72 & $0^{\prime} 50^{\prime \prime}$ & $1^{\prime} 10^{\prime \prime}$ & $2^{\prime} 15^{\prime \prime}$ & 1,83 \\
\hline MI02 & 3,27 & 6,41 & 22,63 & 12,80 & $1^{\prime} 10^{\prime \prime}$ & $1^{\prime} 35^{\prime \prime}$ & $2^{\prime} 10^{\prime \prime}$ & 1,85 \\
\hline MI03 & 2,87 & 4,70 & 29,23 & 22,92 & $1^{\prime} 40^{\prime \prime}$ & $2^{\prime} 10^{\prime \prime}$ & $2^{\prime} 25^{\prime \prime}$ & 1,95 \\
\hline MI04 & 5,20 & 4,74 & 24,66 & 5,82 & $1^{\prime} 23^{\prime \prime}$ & $2^{\prime} 05^{\prime \prime}$ & $2^{\prime} 26^{\prime \prime}$ & 1,35 \\
\hline MI05 & 7,88 & 3,79 & 26,67 & 20,75 & $0^{\prime} 40^{\prime \prime}$ & $1^{\prime} 00^{\prime \prime}$ & $1^{\prime} 35^{\prime \prime}$ & 1,27 \\
\hline MI06 & 6,37 & 6,63 & 24,87 & 11,37 & $0^{\prime} 40^{\prime \prime}$ & $1^{\prime} 05^{\prime \prime}$ & $1^{\prime} 32$ & 1,07 \\
\hline MA01 & 3,88 & 4,74 & 28,55 & 21,17 & $2^{\prime} 30^{\prime \prime}$ & $3^{\prime} 00^{\prime \prime}$ & $3^{\prime} 10^{\prime \prime}$ & 2,33 \\
\hline MA02 & 3,27 & 6,41 & 25,06 & 7,16 & $2^{\prime} 10^{\prime \prime}$ & $2^{\prime} 30^{\prime \prime}$ & $2^{\prime} 40^{\prime \prime}$ & 2,17 \\
\hline MA03 & 2,87 & 4,74 & 16,50 & 6,51 & $1^{\prime} 30^{\prime \prime}$ & $2^{\prime} 10^{\prime \prime}$ & $2^{\prime} 30^{\prime \prime}$ & 2,01 \\
\hline MA04 & 6,40 & 9,24 & 20,35 & 8,68 & $1^{\prime} 21^{\prime \prime}$ & $2^{\prime} 45^{\prime \prime}$ & $3^{\prime} 50^{\prime \prime}$ & 2,52 \\
\hline MA05 & 7,08 & 11,88 & 19,83 & 14,25 & $1^{\prime} 22^{\prime \prime}$ & $3^{\prime} 50^{\prime \prime}$ & $4^{\prime} 33^{\prime \prime}$ & 2,00 \\
\hline MA06 & 11,67 & 12,12 & 40,25 & 23,63 & $1^{\prime} 30^{\prime \prime}$ & $2^{\prime} 40^{\prime \prime}$ & $3^{\prime} 00^{\prime \prime}$ & 2,50 \\
\hline Media & 7,56 & 12,78 & 25,72 & 13,4 & $1^{\prime} 13^{\prime \prime}$ & $2^{\prime} 02^{\prime \prime}$ & $2^{\prime} 30^{\prime \prime}$ & 1,90 \\
\hline
\end{tabular}

La humedad previa de los suelos es muy baja, a pesar de ser la época de lluvias. En superficie (0-2 cm) la humedad es muy baja (7,6\%) mientras que en profundidad (4$6 \mathrm{~cm}$ ) alcanza el 12,78\% del peso del suelo. Tras los experimentos se comprueba que los primeros centímetros almacenan gran cantidad de agua $(25,72 \%)$, mientras que en profundidad no se produce ningún cambio, midiéndose un aumento insignificante en valores medios (13,4\%). Esto indica que después de 45 minutos y $37,8 \mathrm{~mm}$ de lluvia los frentes de humedad no han alcanzado los $4 \mathrm{~cm}$ de profundidad. Un análisis individualizado demuestra que sólo en cuatro de los casos se han superado los $4 \mathrm{~cm}$ de una forma evidente: MI03, MI05, MA01 y MA06. En general, los frentes de humedad han sido muy homogéneos y poco profundos. 
A pesar del reducido contenido en agua de los suelos la escorrentía se genera muy rápidamente. El encharcamiento (tp) del $40 \%$ de la parcela se produce en 1' 13", después de poco más de $1 \mathrm{~mm}$ de lluvia, aunque varía entre $40^{\prime \prime}$ y $2^{\prime} 30^{\prime \prime}$. El inicio de la escorrentía (ts) está claramente influido por el encharcamiento, produciéndose casi un minuto más tarde que éste. El inicio de la escorrentía también es muy variable espacialmente -de 1' a 3' 50"- dependiendo básicamente de la existencia de costras. Tanto las costras de líquenes como las generadas por procesos físicos o químicos favorecen la generación de la escorrentía. También las superficies de las piedras generan abundante y rápidamente escorrentía, aunque parte de ésta es infiltrada en la zona de contracto entre la matriz del suelo y las piedras.

El inicio de la escorrentía en el colector (te) se produce unos 30 segundos después que la escorrentía en la superficie. Este retraso tan reducido se debe a la inexistencia de hojarasca, la reducida rugosidad de las superficies y el encostramiento. Obviamente "tc" también es muy variable temporalmente: entre 1' 32" y 4' 33". Esto significa que la generación de escorrentía necesita muy poco tiempo cuando la intensidad de la lluvia es muy elevada $\left(50 \mathrm{~mm} \mathrm{~h}^{-1}\right)$. Con reducidos volúmenes de lluvia se produce escorrentía superficial $(1,7 \mathrm{~mm})$ y su medición en el colector $(1,9 \mathrm{~mm})$ (Vte).

La escorrentía media es muy elevada en todas las parcelas: $38 \mathrm{~mm} \mathrm{~h}^{-1}$ por término medio, aunque varía entre 45,31 y $23,14 \mathrm{~mm} \mathrm{~h}^{-1}$. Esto es debido a que las tasas de infiltración son extremadamente reducidas $\left(7 \mathrm{~mm} \mathrm{~h}^{-1}\right.$ de media), a que la escorrentía se inicia muy pronto (minuto 2,3 ) y a que las curvas de infiltración tienen unas pendientes muy pronunciadas, es decir, alcanzan la máxima tasa de escorrentía en pocos minutos (ver figuras 6 y 7). Todo esto da lugar a que los volúmenes de agua exportada sean del $79 \%$ en términos medios, variando el coeficiente de escorrentía entre 48 y $94 \%$. Un ejemplo de la rapidez de respuesta de estos suelos ante la lluvia simulada es que el valor del exponente de la ecuación de Horton es, en términos medios, de $0,73 \mathrm{~min} \mathrm{~h}^{-1}$ (ver tabla 3), lo que muestra las fuertes pendientes de las curvas de escorrentía.

Incluso en periodos más reducidos (30 minutos, Rc30) las pérdidas de agua son muy altas: $75 \%$ de la lluvia. Puntualmente encontramos superficies que generan escorrentías cercanas al $90 \%$ de la lluvia para este mismo intervalo.

Las tasas de infiltración son muy reducidas. Hasta el minuto 30 (Vi60) sólo se infiltran $6,26 \mathrm{~mm}$ por término medio. Una proyección de las curvas de infiltración hasta el minuto 60 confirma unas menores tasas de infiltración en los 30 minutos siguientes, ya que la infiltración total (Vi60) no alcanza lo $10 \mathrm{~mm}$. 


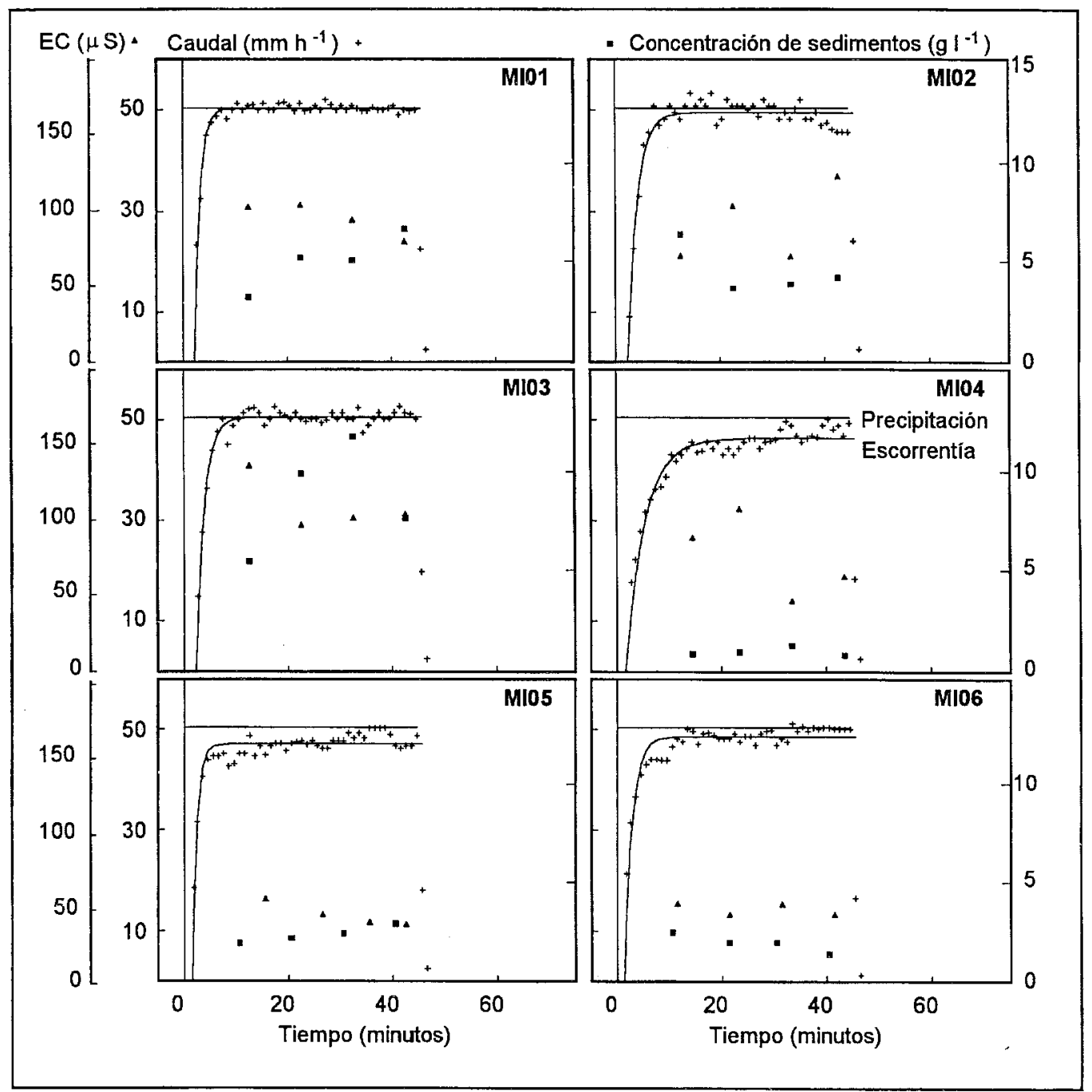

Figura 6.- Hidrogramas de los seis experimentos con lluvia simulada en Mishor Adumin. Escorrentía y precipitación $\left(\mathrm{mm} \mathrm{h}^{-1}\right)$, concentración de sedimentos $\left(\mathrm{g}^{-1}\right)$ y evolución de la conductividad eléctrica de la escorrentía (EC) ( $\mu s)$. 


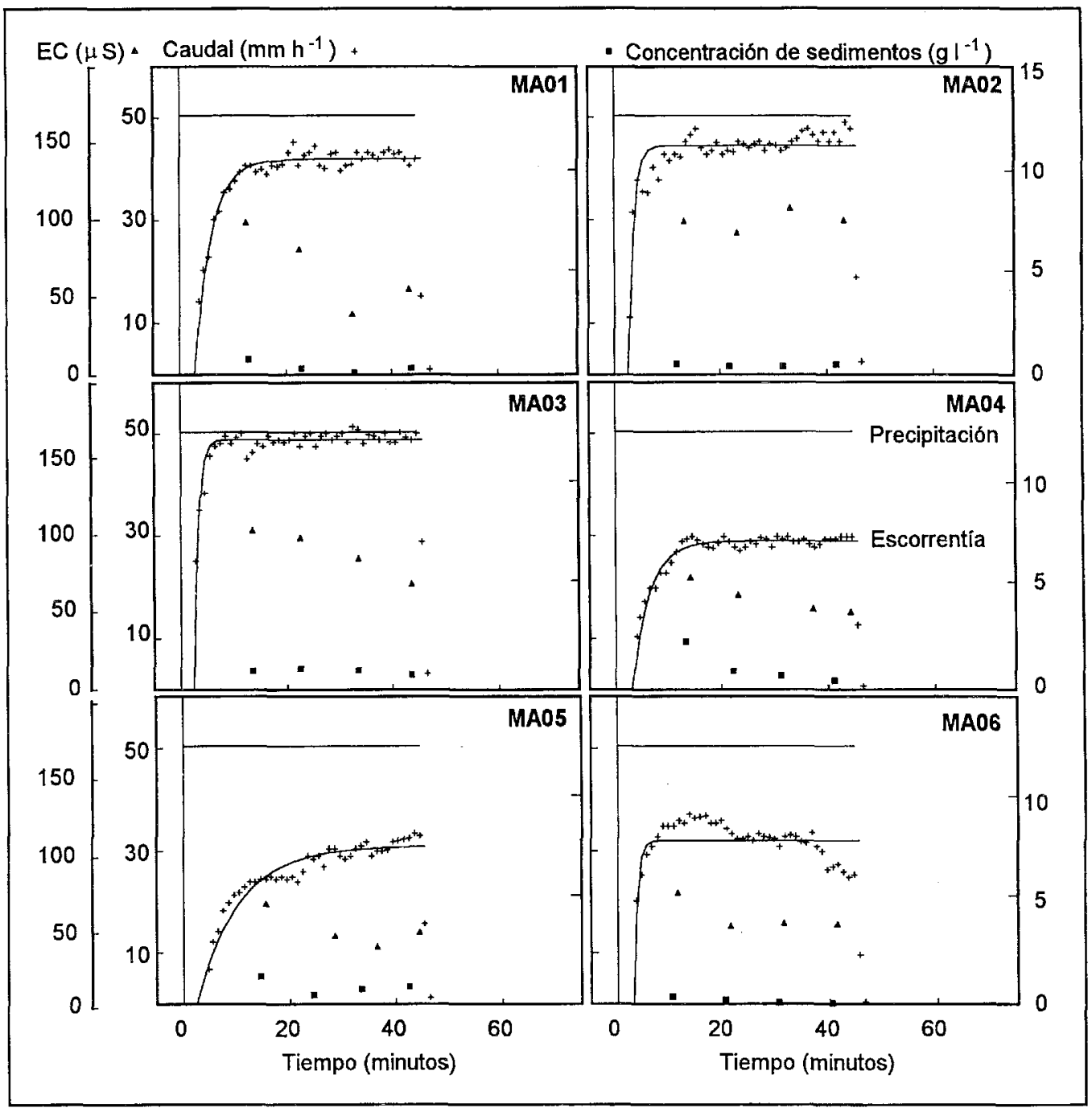

Figura 7.- Hidrogramas de los seis experimentos de lluvia simulada en Ma'ale Adumin. Escorrentía y precipitación $\left(\mathrm{mm} \mathrm{h}^{-1}\right)$, concentración de sedimentos $\left(\mathrm{g} \mathrm{l}^{-1}\right)$ y evolución de la conductividad eléctrica de la escorrentía (EC $(\mu s)$. 
Tabla 3.- Parámetros hidrológicos. Escorrentía media (Ar), Coeficiente de escorrentía (Rc), exponente de la ecuación de Horton, ( $\alpha$, pendiente de la curva de infiltración), tasa de infiltración final estable (fc), volumen de lluvia infiltrada (Vi) a diferentes escalas temporales: hasta el minuto $30(\mathrm{Vi30})$ y hasta el minuto 60 (Vi60), y coeficiente de escorrentía hasta el minuto 30 (Rc30).

\begin{tabular}{|c|c|c|c|c|c|c|c|}
\hline PARCELAS & Ar & Rc & $\alpha$ & fc & Vi30 & Vi60 & Rc30 \\
\hline & mmh $^{-1}$ & $(\%)$ & $\mathrm{min}^{-1}$ & $\mathrm{~mm} \mathrm{~h}^{-1}$ & $\mathrm{~mm}$ & $\mathrm{~mm}$ & $(\%)$ \\
\hline MI01 & 45,31 & 94 & 0,97 & 0,07 & 2,32 & 2,36 & 91 \\
\hline MI02 & 43,80 & 91 & 0,54 & 0,84 & 3,36 & 3,79 & 87 \\
\hline MI03 & 44,74 & 93 & 0,65 & $-0,14$ & 2,75 & 2,68 & 89 \\
\hline M104 & 39,67 & 82 & 0,27 & 3,97 & 5,69 & 7,68 & 77 \\
\hline M105 & 43,09 & 89 & 1,18 & 3,34 & 3,09 & 4,76 & 88 \\
\hline MI06 & 44,03 & 91 & 0,68 & 1,81 & 2,72 & 3,62 & 89 \\
\hline MA01 & 35,32 & 73 & 0,32 & 8,41 & 7,99 & 12,19 & 68 \\
\hline MA02 & 39,64 & 82 & 0,97 & 5,77 & 5,17 & 8,06 & 79 \\
\hline MA03 & 44,08 & 91 & 1,18 & 1,50 & 2,96 & 3,71 & 88 \\
\hline MA04 & 24,12 & 50 & 0,31 & 21,41 & 13,50 & 24,21 & 46 \\
\hline MA05 & 23,14 & 48 & 0,13 & 19,39 & 14,64 & 24,45 & 42 \\
\hline MA06 & 28,70 & 60 & 1,51 & 18,39 & 10,93 & 20,12 & 57 \\
\hline Media & 37,97 & 78,67 & 0,73 & 7,06 & 6,26 & 9,8 & 75,08 \\
\hline
\end{tabular}

Debido a la cubierta de piedras y a las costras físico-químicas y biológicas, la concentración de sedimentos de la escorrentía es muy baja en las parcelas de Ma'ale Adumin (MA) $\left(0,18-0,91 \mathrm{~g} \mathrm{l}^{-1}\right)$. En cambio, en Mishor Adumin (MI) las concentraciones de sedimentos son mucho mayores $\left(0,99-9,42 \mathrm{~g} \mathrm{l}^{-1}\right)$. Esto, junto a los distintos volúmenes de agua exportados da lugar a que en el primer caso la producción de sedimentos sea mucho mayor (1-6 gramos) que en el segundo (7-79 gramos) después de 45 minutos y 37,8 $\mathrm{mm}$ de lluvia (tabla 4 ).

También la pérdida de suelo es muy elevada en Mishor Adumin (5-33 g m-1 h-1) mientras que es mucho más reducida en el caso de Ma'ale Adumin (39-422 g m-1 h ${ }^{-1}$ ). La tasa de erosión media de las 12 mediciones realizadas es de casi $100 \mathrm{~g} \mathrm{~m}^{-1} \mathrm{~h}^{-1}$. La 
concentración de sedimentos decrece con el tiempo en algunos casos. Esto se produce en Ma'ale Adumin en todas las parcelas, excepto en una de ellas donde no hay decrecimiento. En cambio, en Mishor Adumin nos encontramos con un aumento de la concentración de sedimentos por término medio de 0,02 .

La tendencia temporal de la conductividad eléctrica de la escorrentía responde de forma semejante en ambas zonas de estudio: reducción $(-0,62$ por término medio). Sólo en dos casos hay un crecimiento (MI02 y MA02) (ver tabla 4 y figura 2). La salinidad de la escorrentía es muy reducida: 73,41 $\mu \mathrm{S}$, variando entre 44 y $110 \mu \mathrm{S}$.

Tabla 4.- Respuesta erosiva. Concentración de sedimentos de la escorrentía (Sc),

Sedimentos totales (Sy), Tasas de erosión (Er), Conductividad eléctrica (EC), Tendencia de la concentración de sedimentos (TSc), y de la conductividad eléctrica de la escorrentía (TEc).

\begin{tabular}{|c|c|c|c|c|c|c|}
\hline PARCELAS & $\mathrm{Sc}$ & $\mathrm{Sy}$ & $\mathrm{Er}$ & $\mathrm{EC}$ & $\mathrm{TSc}$ & TEc \\
\hline & $\mathrm{g} \mathrm{l}^{-1}$ & $\mathrm{~g}$ & $\mathrm{gm}^{2} \mathrm{~h}^{-1}$ & $\mu \mathrm{S}$ & & \\
\hline MI01 & 5,05 & 42,98 & 228,75 & 95,24 & 0,10 & $-0,78$ \\
\hline MI02 & 4,55 & 36,87 & 199,15 & 91,80 & $-0,06$ & 1,17 \\
\hline MI03 & 9,42 & 79,06 & 421,53 & 109,58 & 0,08 & $-0,92$ \\
\hline MI04 & 0,99 & 7,30 & 39,43 & 76,45 & 0,00 & $-1,43$ \\
\hline MI05 & 2,31 & 18,63 & 99,48 & 43,70 & 0,03 & $-0,63$ \\
\hline MI06 & 1,90 & 15,74 & 83,83 & 47,63 & $-0,03$ & $-0,15$ \\
\hline MA01 & 0,39 & 2,53 & 13,69 & 68,65 & $-0,01$ & $-1,69$ \\
\hline MA02 & 0,46 & 3,41 & 18,26 & 99,38 & 0,00 & 0,18 \\
\hline MA03 & 0,74 & 6,11 & 32,52 & 86,28 & $-0,01$ & $-1,17$ \\
\hline MA04 & 0,91 & 4,11 & 22,06 & 56,73 & $-0,06$ & $-0,72$ \\
\hline MA05 & 0,85 & 3,64 & 19,66 & 48,48 & $-0,01$ & $-0,72$ \\
\hline MA06 & 0,18 & 0,95 & 5,11 & 56,98 & $-0,01$ & $-0,61$ \\
\hline Media & 2,31 & 18,44 & 98,62 & 73,41 & 0,0 & $-0,62$ \\
\hline
\end{tabular}




\section{DISCUSION Y CONCLUSIONES}

En los suelos del desierto de Judea las lluvias intensas, como son las simuladas en este trabajo, generan un encharcamiento muy rápido (1' $\left.13^{\prime \prime}\right)$ y escorrentías casi instantáneas ( $\left.2^{\prime} 02^{\prime \prime}\right)$. Lo anterior, junto a las bajas tasas finales estables de infiltración $\left(7 \mathrm{~mm} \mathrm{~h}^{-1}\right)$, las cuales se alcanzan rápidamente, dan lugar a curvas de escorrentía de gran pendiente y a coeficientes de escorrentía muy elevados. A la escala de la medición realizada ( $55 \mathrm{~cm}$ de longitud) casi el $80 \%$ de la lluvia es transformada en caudal. Los volúmenes infiltrados son insignificantes después de 30 minutos $(6,3$ $\mathrm{mm}$ ) o una hora $(9,8 \mathrm{~mm})$, lo que explica frentes de humedad que normalmente no alcanzan los $4 \mathrm{~cm}$ de profundidad. Los importantes caudales y la reducida protección del suelo por parte de la vegetación generan tasas de erosión bastante elevadas (100 g $\left.\mathrm{m} 2 \mathrm{~h}^{-1}\right)$.

A pesar de estar en el mismo espacio geográfico, entre las dos zonas estudiadas, existen evidentes diferencias. En primer lugar, los condicionantes, aunque semejantes son ligeramente distintos. Por una parte la cubierta de plantas es menor al $2 \%$ en Mishor Adumin, mientras que en Ma'ale Adumin alcanza casi el $10 \%$. En el primer caso, la costra biológica está dominada por los líquenes, y en el segundo por los musgos. Además en Mishor Adumin la presión del pastoreo es mayor, lo que ha favorecido la existencia de costras de origen físico-químico, y a su vez la alteración de éstas por el pisoteo.

Las dos zonas muestran cambios semejantes en la humedad del suelo ante los chaparrones de lluvia: reducida penetración del frente de humedad, y por lo tanto un incremento en la humedad restringido a los primeros centímetros del suelo. A pesar de ello, los cambios sí son apreciables en cuanto a la respuesta hidrológica. En primer lugar, el encharcamiento, el inicio de la escorrentía y la salida de ésta se produce antes en el caso de Mishor Adumin que en Ma'ale Adumin. Los valores medios para cada uno de los parámetros citados son los siguientes: 0' 50", 1' 24", 1' 54" para Mishor Adumin y 1' 26", 2' 37" y 3,06" en Ma'ale Adumin. Esto supone que 1,5 mm de lluvia generan escorrentía en el primer caso, mientras que en el segundo son necesarios hasta 2,3 mm. Ello implica que en Mishor Adumin la escorrentía se generará más frecuente y rápidamente que en Ma'ale Adumin.

Lo anterior explica las mayores tasas de escorrentía en Mishor Adumin. En esta zona los coeficientes de escorrentía son muy elevados: el $90 \%$ de la lluvia se transforma en caudal. En Ma'ale Adumin, también los coeficientes de escorrentía son muy altos, pero menos del $70 \%$ de la lluvia escurre tras 45 minutos a una intensidad de $50 \mathrm{~mm} \mathrm{~h}^{-1}$. Esto supone que después de una hora de lluvia en Mishor Adumin poco más de $4 \mathrm{~mm}$ son infiltrados mientras que $42 \mathrm{~mm}$ son perdidos por escorrentía superficial. En 
Ma'ale Adumin, las cifras son ligeramente distintas. La infiltración supera los $15 \mathrm{~mm}$ mientras que la escorrentía es inferior a $35 \mathrm{~mm}$. Además de la menor aptitud de los suelos de Mishor Adumin para la infiltración, estos son más erosionables. La concentración de sedimentos en la escorrentía varía entre 0,99 y 9,42 $\mathrm{g} \mathrm{l}^{-1}$, siendo la media de 4,04 $\mathrm{g} \mathrm{l}^{-1}$ : En Ma'ale Adumin, siempre es menor a $0,91 \mathrm{~g} \mathrm{l}^{-1}$, mientras que la media no alcanza los $0,6 \mathrm{~g}^{-1}$.

La menor erosionabilidad y la mayor capacidad de infiltración de los suelos de Ma'ale Adumin da lugar a que las tasas de erosión sean muy bajas, $18,55 \mathrm{~g} \mathrm{~m}^{2} \mathrm{~h}^{-1}$, y siempre sean menores a 32,52 $\mathrm{g} \mathrm{m}^{2} \mathrm{~h}^{-1}$. En Mishor Adumin la tasa de erosión media es de $179 \mathrm{~g} \mathrm{~m}^{2} \mathrm{~h}^{-1}$, superando $\operatorname{los} 420 \mathrm{~g} \mathrm{~m}^{2} \mathrm{~h}^{-1}$ en una de las parcelas. La salinidad de las escorrentías, aunque reducida, es mayor en Mishor Adumin (78 $\mu \mathrm{S})$ que en Ma'ale Adumin $(69 \mu \mathrm{S})$. Es destacable la baja salinidad de la escorrentía en comparación con la elevada salinidad de los suelos.

La evolución de la concentración de sedimentos es negativa en Ma'ale Adumin $(<0,02)$ mientras que es positiva en Mishor Adumin $(>0,02)$. Esto indica que en el primero de los casos los sedimentos susceptibles de ser erosionados se agotan rápidamente, mientras que en Mishor Adumin no se agotan, aumentando la concentración de sedimentos con el tiempo. La tendencia de la conductividad eléctrica de la escorrentía es normalmente negativa, lo que se debe al lavado y agotamiento de las sales.

Estas diferencias en el comportamiento de ambas zonas estudiadas se debe en parte a la mayor aridez de Mishor Adumin pero sobre todo, a la mayor presión del pastoreo, el cual degrada las costras superficiales, reduce la cubierta vegetal, altera las superficies de los suelos y con ello favorece la generación de escorrentía superficial. Además, esto supone mayores concentraciones de sedimentos y tasas de erosión en Mishor Adumin que en Ma'ale Adumin. En este último caso, el suministro de sedimentos a la escorrentía por parte del suelo es muy grande debido a la rotura de las costras, como lo demuestra el aumento de la concentración de sedimento con el tiempo. Los sedimentos generados por el paso del ganado, al romper las costras superficiales y movilizar las piedras, junto a la importante deposición de polvo atmosférico explican este comportamiento.

En conclusión, podemos decir que los suelos del Desierto de Judea presentan unas elevadas tasas de escorrentía, que ésta se produce muy rápidamente, y que las curvas de infiltración, además de bajos valores de tasa final estable, presentan curvas de elevadas pendientes. En los casos en los que la presión ganadera es menor y las precipitaciones anuales mayores -Ma'ale Adumin- las tasas de erosión no son muy elevadas porque la pedregosidad y las costras protegen el suelo, lo que da lugar a bajas concentraciones de sedimentos y sales en la escorrentía. En Mishor Adumin, donde el 
sobrepastoreo es evidente, las costras están alteradas, los depósitos de polvo son importantes y la cubierta vegetal es menor; por todo, se produce un aumento de la concentración de sedimentos y los coeficientes de escorrentía, y con ello de las tasas de erosión.

Los experimentos aquí presentados demuestran que la escasa vegetación juega un importante papel en los procesos geomorfológicos debido al control que ejerce sobre la infiltración de las aguas y la erosionabilidad de los suelos (THORNES, 1985; CERDA, 1995), siendo muy importante el control de las costras biológicas (ALEXANDER \& CALVO, 1990). También la pedregosidad superficial determina los procesos hidrológicos, ya que normalmente reduce los caudales (EVENARI et al., 1968; POESEN \& INGELMO SANCHEZ, 1992), si bien en los casos estudiados es la costra superficial, tanto física, biológica como química, la que determina las elevadas tasas de escorrentía, y su destrucción por el sobrepastoreo las altas tasas de erosión. Es por lo tanto el uso del suelo, en este caso el pastoreo, el que explica la respuesta hidrológica y erosiva de los suelos.

En el desierto de Judea, lluvias cortas pero intensas, típicas de los desiertos, producirán escorrentías directas a los cauces (HORTON, 1933), a pesar de que otros mecanismos de generación de escorrentía se puedan producir con intensidades de lluvia menores (YAIR \& LAVEE, 1985), como lo demuestra la existencia de flujos subsuperficiales (LAVEE et al., 1989).

Estudios en los que se incorporen distintas escalas y niveles de precipitación serán necesarios para entender el funcionamiento de los sistemas geomórficos globalmente. En este trabajo, a escala de $55 \mathrm{~cm}$, se ha comprobado que la respuesta de los suelos del desierto de Judea ante lluvias intensas se caracteriza por las abundantes escorrentías generadas, así como por la elevada variabilidad espacial y temporal típica de los ecosistemas semiáridos (LAVEE \& YAIR, 1990).

\section{AGRADECIMIENTOS}

Agradecemos la colaboración de todos los componentes del Laboratory for Geomorphology and Soils de la Bar-Ilan University, en especial O. Ackermann, S Pariente, H. Zhevelev, A. Fragin y T. Svorai. El soporte financiero ha sido aportado parcialmente por el proyecto ERMES (Commission of the European Communities: Environment, 1990-1994, EV5VCT91-0023) y la Conselleria de Educació i Ciència de la Comunitat Valenciana. La redacción del trabajo ha sido posible por la concesión de una beca de Perfeccionamiento de Doctores y Tecnólogos en el Extranjero por el Ministerio de Educación y Ciencia al primero de los autores. 


\section{BIBLIOGRAFIA}

ALEXANDER, R.W. \& CALVO, A. (1990): The influence of lichens on slope processes in some Spanish Badlands, En: THORNES, J.B. (Ed.): Vegetation and Erosion: Process and Environments, 385-98.

CERDA, A. (1993a): La Infiltración en los Suelos del País Valenciano. Factores y Variaciones Espacio-Temporales. Tesis Doctoral Inédita, Universitat de València, $357 \mathrm{p}+$ apéndices.

CERDA, A. (1993b): Metodología para el estudio de la hidrología y erosión de superficies degradadas (badland) a partir de lluvia simulada", Cuaternario y Geomorfología, 7.

CERDA, A. (1995): Factores y Variaciones Espacio-Temporales de la Infiltración en los Ecosistemas Mediterráneos. Editorial Geoforma, 159 p.

CERDA, A. y LAVEE, H. (1994): Respuesta a la lluvia simulada de los suelos del Desierto de Judea, Kalia, Israel. En: ARNAEZ, J. GARCIA RUIZ, J.M. y GOMEZ VILLAR, A. (Eds.): Geomorfología en España, 133-142.

CERDA, A. \& LAVEE, H. (en prensa): The impact of grazing on erosion processes along an altitudinal transect in Israel, En: Oostwoud, D. y Cerdá, A. (Eds.) Dynamical Geomorphology of Slopes and River Beds in Alpine Catchments: the Dilemma of Climatic Change.

COOKE, R.U. \& REEVES, R.W. (1972): Geomorphology in Deserts. London, Batsford.

COOKE, R.U., WARREN, A. \& GOUDIE, A. S. (1993): Desert Geomorphology. UCL Press, London.

EVENARI, M.L., SHANAN, L. \& TADMOR, N. (1968): Runoff farming in the desert. (I), Experimental layout. Agronomy Journal, 60, 29-32.

HORTON, R.E. (1933): The role of infiltration in the hydrologic cycle. EOS Trans A.G.U., 14, 446460.

HORTON, R.E. (1940): An approach toward a physical interpretation of infiltration capacity. Proc. Soil Sci. Soc. Am., 5, 399-417.

IMESON, A.C. \& VERSTRATEN, J.M. (1988): Rills on badland slopes: a physico-chemical controlled phenomenon. Catena supplement 12, 139-50.

LAVEE, H. \& YAIR, A. (1990): Spatial Variability of Overland Flow in a Small Arid Basin. IAHS Publication, 189, 185-120. 
LAVEE, H., IMESON, A.C., PARIENTE, S. \& BENYAMINI, Y. (1991): The response of soils to simulated rainfall along a climatological gradient in an arid and semiarid region" Catena Supplement, 19, 19-37.

LAVEE, H., PARIENTE, S., ZHEVELEV, H., FRAGIN, A., \& SVORAI, T. (1994): Final Report ERMES ECC Research Project: Modelling and exploring the impact of climatic change on ecosystem degradation, hydrology and land use along a transect across the Mediterranean. Bar-Ilan University, Laboratory for Geomorphology and Soils, Ramat-Gan, Israel, 38 p.

LAVEE, H., WIEDER, M. \& PARIENTE, S. (1989): Pedogenic Indicators of Subsurface Flow on Judean Desert Hillslopes. Earth Surface Processes and Landforms, 14, 545-555.

LEOPOLD, L.B., EMMETT, W.W \& MYRICK, R.M. (1966): Channel and hillslope processes in a semiarid area. New Mexico. USGS Professional Paper 352G.

POESEN, J. \& INGELMO-SANCHEZ, F. (1992). Runoff and sediment yields from topsoils with different porosity as affected by rock fragment cover and position" Catena, 19.

SCHICK, A.P. (1977): A tentative sediment butget for an extremely arid watershed in the southern Negev. En: DOEHRING, D.O. (Ed.): Geomorphology in arid regions, 139-161.

SCHICK, A.P. (1987): Hydrologic aspects of floods in extreme arid environments. En: BAKER, V.R., KOCHEL, R.C. \& PATTON, P.C. (Eds.): Flood geomorphology, 189-203.

SHAINBERG, I., ROADES, J.D. \& PRATHER, R.J. (1981): Effect of low electrolyte concentration on clay dispersion and hydraulic conductivity of a sodic soil. Soil Sci. Soc. Am. J., 45, 273-277)

SLAYTER, R.O. \& MABBUTT, J.A. (1964): Hydrology of arid and semiarid regions. En: CHOW (Ed.): Handbook of applied hydrology, 24-26.

THORNES, J.B. (1985): The ecology of erosion" Geography, 70 (3), 222-36.

THORNES, J.B. (1994): Catchment and Channel Hydrology. En: ABRAHAMS, A.D. \& PARSONS, A.J. (Eds.): Geomorphology of Desert environments, 257-287.

YAIR, A. \& LAVEE, H. (1985): Runoff generation in arid and semi-arid zones. En ANDERSON \& BURT, T.P. (Eds.): Hydrological Forecasting, 183-220. 